\title{
World Health Organization Integrated Care for Older People (ICOPE) and the Integrated Care of Older Patients with Frailty in Primary Care (ICOOP Frail) Study in Korea
}

\author{
Chang Won Won ${ }^{1}$, Eunmi Ha ${ }^{2}$, Eunjin Jeong ${ }^{1}$, Miji Kim ${ }^{3}$, Juhyun Park, Ja Euk Baek ${ }^{5}$, Sungouk Kim ${ }^{6}$, Sung Bae Kim², Jihun Roh ${ }^{8}$, \\ Jee Hye Choi ${ }^{9}$, Seung Youn Jeon ${ }^{10}$, Heeeun Jung ${ }^{2}$, Daehyun Lee ${ }^{2}$, Yuri Seo ${ }^{2}$, Hyungeun Shin ${ }^{2}$, Heesun Kim ${ }^{4}$ \\ ${ }^{1}$ Department of Family medicine, Kyung Hee University Medical Center, College of Medicine, Kyung Hee University, Seoul, Korea \\ ${ }^{2}$ Department of Biomedical Science and Technology, Graduate School, Kyung Hee University, Seoul, Korea \\ ${ }^{3}$ Department of Biomedical Science and Technology, College of Medicine, East-West Medical Research Institute, Kyung Hee University, Seoul, Korea \\ ${ }^{4}$ National Evidence-based Healthcare Collaborating Agency, Seoul, Korea \\ ${ }^{5}$ Department of Family Medicine, Dongdong Family Medicine Clinic, Seoul, Korea \\ ${ }^{6}$ Department of Surgery, Dongbu-hanil Surgery Clinic, Seoul, Korea \\ ${ }^{7}$ Department of Family Medicine, Mirae Family Medicine Clinic, Seoul, Korea \\ ${ }^{8}$ Department of Family Medicine, Seoul Family Medicine Clinic, Seoul, Korea \\ ${ }^{9}$ Department of Family Medicine, Woori Family Medicine Clinic, Seoul, Korea \\ ${ }^{10}$ Department of Family Medicine, Haneul Family Medicine Clinic, Uijeongbu, Korea
}

\section{Corresponding Author:}

Heesun Kim, MD, $\mathrm{PhD}$

Department of Health Policy Research, National Evidence-based Healthcare Collaborating Agency, Namsan Square (Kukdong B/D) 7F, 173 Toegye-ro, Jung-gu, Seoul 04554, Korea

E-mail: hskim7336@neca.re.kr ORCID:

https://orcid.org/0000-0003-4836-907X

Received: February 27, 2021

Revised: March 19, 2021

Accepted: March 19, 2021
The World Health Organization (WHO) recently endorsed the proposal for a Decade of Healthy Ageing (2020-2030). The WHO defines "healthy aging" as "the process of developing and maintaining the functional ability that enables wellbeing in older age." Among the strategies for the Decade of Healthy Ageing, the WHO has suggested enhancing intrinsic capacity, promoting functional ability, and implementing the Integrated Care for Older People (ICOPE) package. The WHO has defined steps for ICOPE evaluation and scale-up and is performing a prospective study in 2-3 countries (low and middle income, high income) to test its feasibility in 2021-2022 and a multinational randomized study to validate its clinical efficacy and effectiveness in 2022-2024. Intrinsic capacity and frailty represent two faces of the same coin, with one indicating the reserves of the individual and the other indicating the deficits that accumulate with age. The Integrated Care of Older Patients with Frailty in Primary Care (ICOOP_Frail) study is the first integrated care program for frailty or functional decline in primary care in Korea. The results suggest that the ICOOP_Frail study can be utilized as a reference for ICOPE studies in Korea or at least to provide important findings for the forthcoming ICOPE implementation study in Korea.

Key Words: Healthy aging, Frailty

\section{INTRODUCTION}

On August 3, 2020, the World Health Assembly of the World Health Organization (WHO) endorsed the proposal for a Decade of Healthy Ageing (2020-2030). Thereafter, the proposal was submitted to the United Nations General Assembly, which proclaimed 2021-2030 as the United Nations (UN) Decade of Healthy Age- ing on December 14, 2020. ${ }^{1)}$ Following the UN decision, governments, international and regional organizations, civil society, the private sector, academia, and the media have been encouraged to actively support the Decade's goals. ${ }^{1)}$

The WHO defines "healthy aging" as "the process of developing and maintaining the functional ability that enables wellbeing in older age." Functional ability is the capability of people to do what 
they have reason to value. Being free of disease is not a requirement for healthy aging as many older adults have one or more health conditions that, when well-controlled, have little influence on their functional abilities. ${ }^{1)}$ By its definition, functional ability includes a person's capacity to: ${ }^{1)}$ meet his or her basic needs; learn, grow, and make decisions; be mobile; build and maintain relationships; and contribute to society. Therefore, "functional ability" depends on the intrinsic capacity (IC) of the individual, relevant environmental characteristics, and the interaction between them. ${ }^{1)}$

The concept of IC was first introduced by the WHO in 2015 to create a multidimensional indicator related to an individual's functional status, the follow-up of which over time may be useful to achieve healthy aging. ${ }^{2)}$ IC comprises all the mental and physical capacities upon which a person relies and includes their ability to walk, think, see, hear, remember, etc. In addition, several factors, including diseases, injuries, and age-related changes, may influence the level of IC. The environment includes the home, community, and broader society and all the factors within them, such as the facilities, people and their relationships, attitudes and values of society members, health and social policies of the government, and the systems and services that support them. ${ }^{1 \text { ) }}$

As one of the strategies to implement the Decade of Healthy Ageing by enhancing IC, promoting functional ability, and minimizing care dependency, the WHO provided the Integrated Care for Older People (ICOPE) package in 2017. Evidence-based programs help key stakeholders in health and social care to understand, design, and implement a person-centered and coordinated model of care. The ICOPE program offers evidence-based tools and guidance specific to every level of care to maximize the IC and functional ability of older people.

Another concept associated with reduced IC is frailty. Frailty is related to functional deficits, and IC refers to functional reserve. Therefore, efforts to diagnose and manage frailty may help assess and prevent declines in IC. The authors recently started a randomized controlled trial of the Integrated Care of Older Patients with Frailty in Primary Care (ICOOP_Frail) to identify how the ICOOP_Frail could contribute to the WHO ICOPE by reviewing the associations between the two programs.

\section{ICOPE GUIDELINES PUBLISHED BY THE WHO}

The current ICOPE guidelines are organized into three modules. ${ }^{3)}$

Module I: Declines in IC, including mobility loss, malnutrition (vitality), visual impairment, hearing loss, cognitive impairment, and depressive symptoms.

Module II: Geriatric syndromes associated with care dependency, including urinary incontinence and risk of falls.

Module III: Caregiver support that includes interventions to support caregiving and prevents caregiver strain.

These guidelines cover evidence-based interventions to manage common declines in capacity in older age, including mobility, nutrition or vitality, vision, hearing, cognition, and mood, as well as important geriatric syndromes of urinary incontinence and risk of falls. These conditions were selected because they are strong independent predictors of mortality and care dependency in older age.

The general care pathways for those functional declines comprise the following five steps. ${ }^{3)}$

Step 1: Screen for declines in IC.

Step 2: Undertake a person-centered assessment in primary care.

Step 3: Define the goal of care and develop a personalized care plan.

Step 4: Ensure a referral pathway and monitor the care plan with links to specialized geriatric care.

Step 5: Engage communities and support caregivers.

IC and frailty represent the two faces of the same coin, with one indicating the reserves of the individual and the other the deficits that accumulate with aging.)

Although both frailty and IC are dynamic entities, frailty is mainly used in cross-sectional assessments, while IC is applied in longitudinal approaches. However, instead of the frailty phenotype, a frailty index can be used in longitudinal approaches. The frailty index is related to physical, psychological, cognitive, sensory, and social functional declines, and the index is used not only to identify frail persons but also those at risk of frailty. ${ }^{5)}$

In addition, the frailty index and IC are based on the assumption that aging individuals can be adequately assessed and managed only if comprehensively evaluated and followed in a novel healthcare model based on service integration and multidisciplinarity. ${ }^{2)}$

Therefore, the integrated care of older patients with frailty or risk of frailty using the frailty index is as important for achieving healthy aging as reducing frailty level is for promoting it. ${ }^{6}$

\section{ICOPE IMPLEMENTATION PILOT PROGRAMS WORLDWIDE}

The first phase of the ICOPE implementation pilot program started in France, Andorra, China, India, Italy, Kenya, Mexico, Qatar, and Vietnam to assess its feasibility and acceptability. ${ }^{3)}$ In France, the ICOPE pilot program (the INSPIRE ICOPE-CARE program) was implemented in clinical practice in the Occitania region to 
promote healthy aging and maintain the autonomy of seniors based on the use of digital medicine. ${ }^{7)}$

The target population is independent seniors aged 60 years and over. The six domains of IC are systematically monitored using pre-established tools proposed by the WHO, which have been adopted in digital form to allow remote and large-scale monitoring.

Step 1 (screening) of the INSPIRE ICOPE-CARE program is performed every 4-6 months by professionals or the seniors themselves. If a deterioration in one or more domains of IC is identified, an alert is generated by an algorithm, which allows rapid intervention by health professionals. The operational implementation of the INSPIRE ICOPE-CARE program in Occitania is performed by the Territorial Teams of Aging and Prevention of Dependency network, which comprises 2,200 professionals in the medical, medico-social, and social sectors. The program aims to screen and monitor 200,000 older people in the Occitania region within 3-5 years and promote preventive actions. The French Presidential Plan Grand Age has largely implemented the WHO ICOPE program in France based on the experiences of the INSPIRE ICOPECARE program in Occitania. ${ }^{7)}$

Step 2 (person-centered assessment) and Step 3 (personalized care plan) of the INSPIRE ICOPE-CARE program are conducted by a general practitioner or if possible by a nurse trained in geriatrics. Experienced trained nurses of the Gerontopole perform geriatric evaluations of older people in their homes or public spaces close to their homes. These nurses with the support of a hospital geriatrician propose personalized care plans for the older people evaluated. This plan is then sent to the general practitioner to ensure its implementation and follow-up. ${ }^{7)}$

The WHO ICOPE's Ready-Set-Go approaches to test and learn from the feasibility and acceptability of pilot studies, as well as studies to evaluate clinical effectiveness and efficacy are planned to be completed by 2024 (Table 1). From 2025, ICOPE will be rolled out within primary healthcare settings in a wider range of countries. $^{3)}$

\section{THE ICOOP_FRAIL STUDY}

Since June 2020, we have been conducting the ICOOP_Frail randomized controlled trial of the National Evidence-based Healthcare Collaborating Agency in Korea. The study is underway in four primary care clinics, and two more clinics have recently joined. Nurses and doctors at the clinics apply the Korean Frailty Index for Primary Care (KFI_PC) to evaluate the functional decline and frailty of older adult patients visiting the clinics. ${ }^{8)}$ The KFI_PC consists of 54 items in the following 10 domains: (1) cognitive status, including delirium or dementia; (2) mood; (3) communication, including vision, hearing, and speech; (4) mobility; (5) balance; (6) bowel function; (7) bladder function; (8) ability to perform activities of daily living; (9) nutrition; and (10) social resources. All items in the KFI_PC are already embedded in and displayed on a mobile notepad so that the nurses and doctors can easily evaluate the patient, determine the frailty index score, and assess risk factors for frailty, which are automatically displayed on the notepad. In the ICOOP_Frail study, patients with KFI_PC scores $\geq 0.25$, which is defined as frail, are randomized into experimental or control groups (Fig. 1).

In the experimental group, primary care physicians explain functional declines or risk factors and educate patients about nutrition or exercise, adjust medication, and manage diseases based on the recommendations automatically displayed for the patient (Table 2). Within 1 month, a health coach contacts the patient via telephone to monitor the patient and encourage their consumption of a protein-rich balanced diet or/and practicing exercise, as well as connects the patient to community resources for social support, if needed. We surveyed the available community resources for social welfare services managed by community administration centers or senior welfare centers in advance to connect the participants to favored services. Unfortunately, all community services have been closed due to the coronavirus disease 2019 (COVID-19) pandemic. The health coaches involved in ICOOP_Frail are paramedical students studying gerontologic epidemiology at Kyung Hee University. In the future, volunteers or public health workers in the community will be welcome to serve as health coaches. The phone

Table 1. Steps for ICOPE evaluation and scale-up: the Ready-Set-Go approach

\begin{tabular}{llll}
\hline Ready (2020-2021) & \multicolumn{1}{c}{ Set (2021-2022) } & Go (2022-2024) & Universal health coverage (2025-2030) \\
\hline $\begin{array}{l}\text { Analysis of real-world usability of } \\
\text { ICOPE Handbook \& Readiness sur- } \\
\text { vey at system and service levels based } \\
\text { on an ICOPE implementation score- } \\
\text { card }\end{array}$ & $\begin{array}{c}\text { Prospective study in 2-3 countries (low Multinational (10+ countries) random- Based on previous results, countries se- } \\
\text { and middle income, high income) to } \\
\text { test the feasibility, identify barriers } \\
\text { and enablers, and refine outcome in- } \\
\text { dicators }\end{array}$ & $\begin{array}{l}\text { ized study to validate the clinical effi- } \\
\text { cacy and effectiveness }\end{array}$ & $\begin{array}{l}\text { lect ICOPE interventions as part of } \\
\text { their national benefit packages }\end{array}$ \\
\hline
\end{tabular}

Referenced by World Health Organization. Guidelines on Integrated Care for Older People (ICOPE). Available from: https://www.who.int/ageing/ publications/guidelines-icope/en/. 


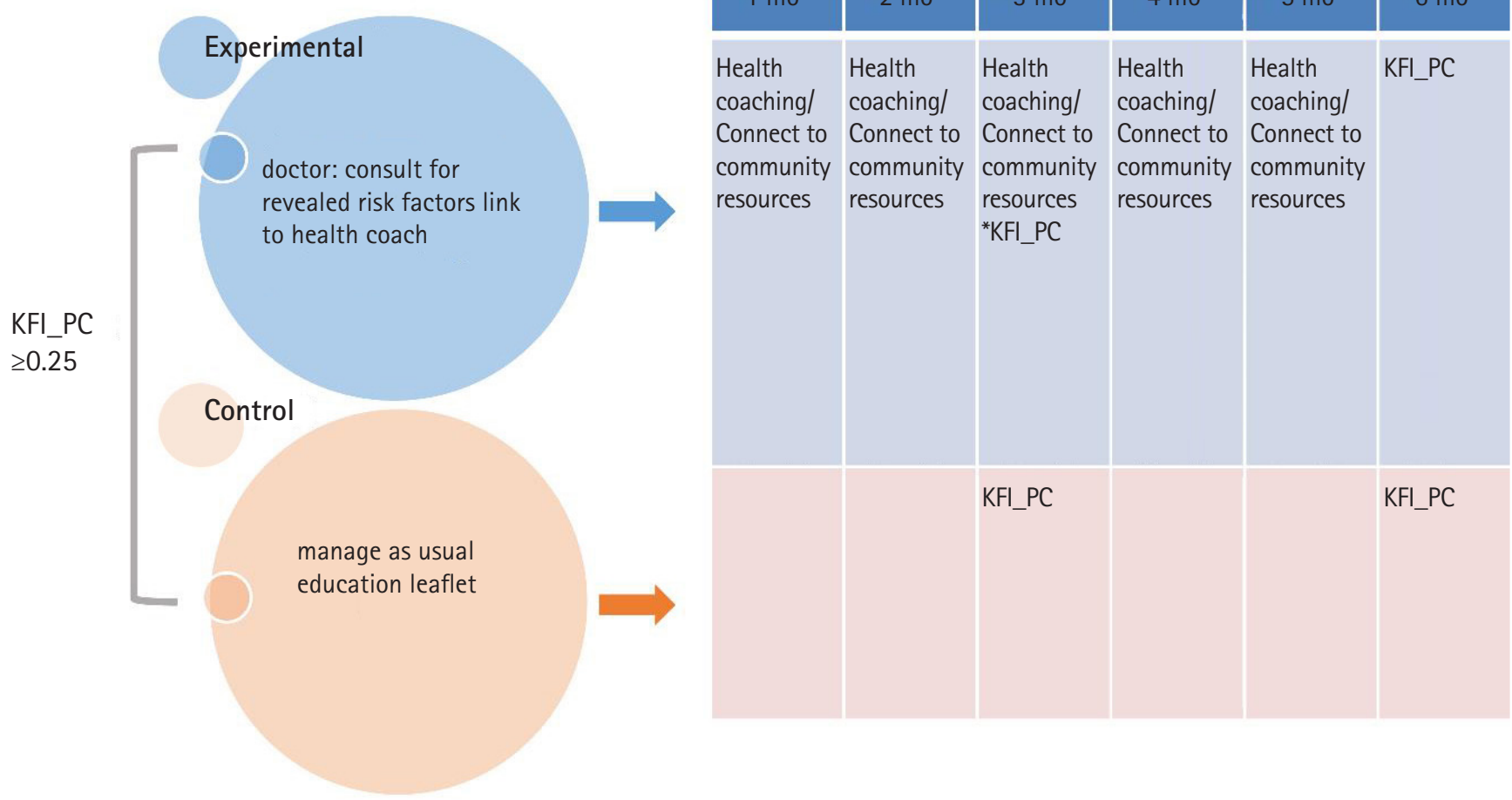

Fig. 1. ICOOP_Frail study, patients with the Korean Frailty Index for Primary Care (KFI_PC) scores $\geq 0.25$, which is defined as frail, are randomized into experimental or control groups.

Table 2. Example of identified frailty risk factors (functional declines) and displayed recommendations in the ICOOP_Frail study

\begin{tabular}{|c|c|}
\hline Identified problem & Recommendations \\
\hline \multirow[t]{3}{*}{ Sarcopenia suspected } & Rule out underlying diseases. \\
\hline & Protein intake of $20 \mathrm{~g}$ per meal. \\
\hline & Resistance exercise twice weekly and aerobic exercise 30 minutes daily. \\
\hline Hearing problem & Refer to ENT doctor. \\
\hline \multirow[t]{4}{*}{ Sleep problems } & Investigate the causes. \\
\hline & Improve the sleep environment and increase daytime outdoor activities. \\
\hline & Stop unnecessary psychotropic use. \\
\hline & Treat depression if indicated. \\
\hline \multirow[t]{3}{*}{ Daytime drowsiness } & Stop unnecessary use of psychotropics or sleep pills. \\
\hline & Investigate the presence of sleep problems at night. \\
\hline & Investigate the presence of snoring during sleep (sleep apnea). \\
\hline \multirow[t]{3}{*}{ Urinary incontinence } & Urinalysis. \\
\hline & Evaluate bladder movement. \\
\hline & Urge incontinence : try bladder-specific anticholinergics or consult a urologist. \\
\hline IADL impairment & Arrange the help of family or caregiver (or care-worker). \\
\hline Insufficient social contact & $\begin{array}{l}\text { Link to nearby community resources or services; search for home visiting service if needed. A health coach may visit the patient's } \\
\text { home. }\end{array}$ \\
\hline \multirow[t]{3}{*}{ Fewer than two meals per day } & Check for the cause of infrequent meals; e.g., economic problems, no person to provide meals, or disease. \\
\hline & Link to nearby community resources or services; search for food delivery services. \\
\hline & A health coach may visit the patient's home. \\
\hline Diseases & Check whether the diseases are well-managed. \\
\hline
\end{tabular}

ICOPE, Integrated Care for Older People; ENT, ear-nose-throat; IADL, instrumental activities of daily living. 
call for health coaching is repeated monthly for a total of 6 months. The KFI_PC is re-evaluated after 3 and 6 months. The primary outcome variables were defined as frailty index and the number of acute medical visits, while activities of daily living, health habits (exercise, diet), social activities, and gait ability were defined as the secondary outcomes.

\section{WHO ICOPE AND ICOOP_FRAIL IN PRIMARY CARE}

IC and frailty represent the two faces of the same coin. One indicates individual reserves, while the other indicates deficits that have accumulated with aging. ${ }^{2)}$ Although both frailty and IC are dynamic entities, frailty is mainly evaluated in cross-sectional assessments, while IC is often used in longitudinal approaches. However, instead of the frailty phenotype, the frailty index can also be used in longitudinal approaches. The frailty index is related to declines in physical, psychological, cognitive, sensory, and social functions and can be used to identify not only frail persons but also those at risk of frailty. ${ }^{5)}$ In addition, the frailty index and IC are based on the assumption that aging individuals can be adequately assessed and managed only if comprehensively evaluated and followed in a novel healthcare model based on service integration and multidisciplinarity. ${ }^{2}$ Therefore, the integrated care of older patients with frailty or risk of frailty based on the frailty index is as important for achieving healthy aging as reducing frailty is for promoting healthy aging. ${ }^{6}$ )
The WHO has described steps to evaluate and scale-up ICOPE, including a prospective study in $2-3$ countries (low and middle income, as well as high income) in 2021-2022 to test its feasibility and a multinational randomized study in 2022-2024 to validate its clinical efficacy and effectiveness. The managed care system in Korea has implemented a chronic disease management program in primary care ${ }^{9)}$ and evaluated a technology-enhanced integrated care model for a frail older person in nursing home; ${ }^{10)}$ however, the ICOOP_Frail study is the first integrated care program for frailty or functional decline in primary care in Korea.

Comparisons of the eight core items of WHO ICOPE and 53 items of the KFI_PC in ICOOP_Frail showed that all the eight core items of the ICOPE were well integrated into the KFI_PC (Table 3). In addition, WHO ICOPE and ICOOP_Frail both (1) are designed to be implemented in primary care at the community level, (2) are integrated programs that consider not only medical but also social and psychological aspects; (3) develop personalized care plans; and (4) aim to promote healthy aging (Table 4). Therefore, we suggest that the ICOOP_Frail study can be utilized as a reference study for ICOPE studies in Korea or may provide important findings for future studies on ICOPE implementation in Korea. However, ICOOP_Frail does not include the referral pathway and caregiver support addressed in ICOPE.

Table 3. Eight core items of the WHO ICOPE and their corresponding items in the Korean Frailty Index for Primary Care (KFI_PC)

\begin{tabular}{|c|c|c|c|}
\hline & & ICOPE & KFI_PC (ICOOP_Frail) \\
\hline \multirow{9}{*}{$\begin{array}{l}\text { Six items of intrinsic } \\
\text { capacity }\end{array}$} & Cognitive decline & Remember three words & Remember three words \\
\hline & & Orientation in time and space & Orientation in time (month, weekday) \\
\hline & & Recall the three words & Recall the three words \\
\hline & Limited mobility & Chair rise test & Chair rise test \\
\hline & Malnutrition & Weight loss & Weight loss of $4.5 \mathrm{~kg}$ or more per year \\
\hline & & Appetite loss & SNAQ question: How is your appetite? \\
\hline & Visual impairment & $\begin{array}{l}\text { Ask patients if they have any problems with their eyes, } \\
\text { such as seeing far away, reading, eye disease or under } \\
\text { medical treatment (diabetes mellitus, hypertension) }\end{array}$ & $\begin{array}{l}\text { Ask patients if they have any problems with their sight in } \\
\text { daily life }\end{array}$ \\
\hline & Hearing loss & Whisper test or audiometry & $\begin{array}{l}\text { Ask patients if they have any problems with their hearing } \\
\text { in daily life }\end{array}$ \\
\hline & Depressive symptoms & $\begin{array}{l}\text { Ask patients if they are feeling down, depressed, or hope- } \\
\text { less }\end{array}$ & $\begin{array}{l}\text { Ask patients if they have felt depressed or sad in the last } \\
\text { month }\end{array}$ \\
\hline \multirow[t]{4}{*}{$\begin{array}{l}\text { Two items related to } \\
\text { geriatric syndromes }\end{array}$} & Urinary incontinence & $\begin{array}{l}\text { Ask patients if they have a strong and sudden urge to void } \\
\text { that makes them leak before reaching the toilet }\end{array}$ & Urinary incontinence experience in the past month \\
\hline & Risk of falls & $\begin{array}{l}\text { Recurrent falls in the past year or gait and/or balance ab- } \\
\text { normalities }\end{array}$ & Frequency of falls in the past year \\
\hline & & & Use of a walking aid \\
\hline & & & Balance problem as evaluated by a physician \\
\hline
\end{tabular}

WHO, World Health Organization; ICOPE, Integrated Care for Older People; SNAQ, Simplified Nutritional Appetite Questionnaire. 
Table 4. Comparisons of WHO ICOPE and ICOOP_Frail: concept, purpose, design, contents, outcomes, and policy implications

\begin{tabular}{|c|c|c|}
\hline & ICOPE & ICOOP_Frail \\
\hline \multirow[t]{3}{*}{ Conceptual framework } & $\begin{array}{l}\text { To reverse or delay declines in intrinsic capacity is crucial for } \\
\text { healthy aging. }\end{array}$ & $\begin{array}{l}\text { Frail people are at higher risks for impaired function, which hinders } \\
\text { healthy aging. }\end{array}$ \\
\hline & $\begin{array}{l}\text { Declines in intrinsic capacity are often not identified, treated, or } \\
\text { monitored. }\end{array}$ & $\begin{array}{l}\text { Frailty is the status of decreased functional reserve (intrinsic capac- } \\
\text { ity). }\end{array}$ \\
\hline & $\begin{array}{l}\text { Most healthcare professionals lack guidance or training to recog- } \\
\text { nize and manage impairments in intrinsic capacity in older adults. }\end{array}$ & $\begin{array}{l}\text { The progression of frailty can be delayed or slowed, which will lead } \\
\text { to healthy aging. }\end{array}$ \\
\hline Purpose & Maximize intrinsic capacities and functional abilities. & $\begin{array}{l}\text { Maximize the intrinsic capacities and manage frailty to maintain } \\
\text { functional abilities. }\end{array}$ \\
\hline \multirow[t]{2}{*}{ Design } & A person-centered and coordinated model & A person-centered and coordinated model \\
\hline & Comprehensive assessment and care plans shared with all provid- & Comprehensive assessment and care plans \\
\hline & $\begin{array}{l}\text { malnutrition, visual impairment, hearing loss, cognitive impair- } \\
\text { ment, and depressive symptoms. }\end{array}$ & 2. Comprehensive geriatric assessment, including a frailty index. \\
\hline & $\begin{array}{l}\text { Module II: Geriatric syndromes associated with care dependency, } \\
\text { including urinary incontinence and risk of falls. }\end{array}$ & $\begin{array}{l}\text { 3. Creation of individualized wellness plans to enhance patient } \\
\text { health, including exercise, socialization, and nutrition. }\end{array}$ \\
\hline & $\begin{array}{l}\text { Module III: Caregiver support: interventions to support caregiving } \\
\text { and prevent caregiver strain. }\end{array}$ & $\begin{array}{l}\text { 4. Coaching: telephone-based health coaching to support patients } \\
\text { and track their progress in achieving goals in terms of exercise, } \\
\text { chronic healthcare issues management, and connections to re- } \\
\text { sources in the community. }\end{array}$ \\
\hline \multirow[t]{3}{*}{ Outcomes } & Maintain intrinsic capacity & Improve frailty index (maintain intrinsic capacity) \\
\hline & Prevent and ensure well-management of existing diseases & Improve functional ability \\
\hline & Optimize functional ability & Increase healthy habits (exercise, diet) \\
\hline
\end{tabular}

WHO, World Health Organization; ICOPE, Integrated Care for Older People.

\section{CONCLUSION}

The WHO recently endorsed the proposal for a Decade of Healthy Aging (2020-2030). The WHO defines "healthy aging" as "the process of developing and maintaining the functional ability that enables wellbeing in older age." Among the strategies to implement the Decade of Healthy Ageing, the WHO suggested the enhancement of intrinsic capacity and use of the ICOPE package program.

ICOOP_Frail is the first integrated care program for frailty or functional decline in primary care in Korea. Comparison of the eight core items of the WHO ICOPE to the 53 items of the KFI PC revealed that all the eight core items were well integrated into the KFI_PC, upon which the ICOOP_Frail study was based.

We suggest that the ICOOP_Frail study can be utilized as a reference study for ICOPE studies in Korea or provide important findings for future ICOPE implementation studies in Korea.

\section{ACKNOWLEDGEMENTS}

This work was supported by National Research Foundation (NRF) grants, funded by the National Evidence-based Healthcare Collaborating Agency (Grant No. NECAIRB20-021-2; Research No. NA20-007). The content is solely the responsibility of the authors and does not necessarily represent the official views of the funding sources.

\section{CONFLICT OF INTEREST}

The researchers claim no conflicts of interest. 


\section{AUTHOR CONTRIBUTION}

Conceptualization, CWW, HK; Supervision, CWW, HK; Writing -original draft, CWW; Writing-review \& editing, EH, JP, JEB, SK, SBK, JR, JHC, SYJ, HJ, DL, YS, HS; CWW, EH, EJ, MK, HK.

\section{REFERENCES}

1. World Health Organization. Ageing: healthy ageing and functional ability [Internet]. Geneva, Switzerland: World Health Organization; c2020 [cited $2021 \mathrm{Mar} 25$ ]. Available from: https://www. who.int/news-room/q-a-detail/ageing-healthy-ageing-and-functional-ability.

2. Belloni G, Cesari M. Frailty and intrinsic capacity: two distinct but related constructs. Front Med (Lausanne) 2019;6:133.

3. World Health Organization. Guidelines on Integrated Care for Older People (ICOPE) [Internet]. Geneva, Switzerland: World Health Organization; 2017 [cited 2021 Mar 25]. Available from: https://www.who.int/ageing/publications/guidelines-icope/ en/.

4. Jotheeswaran AT, Bryce R, Prina M, Acosta D, Ferri CP, Guerra $\mathrm{M}$, et al. Frailty and the prediction of dependence and mortality in low- and middle-income countries: a 10/66 population-based cohort study. BMC Med 2015;13:138.

5. Won CW. Diagnosis and management of frailty in primary health care. Korean J Fam Med 2020;41:207-13.

6. Nwagwu VC, Cigolle C, Suh T. Reducing frailty to promote healthy aging. Clin Geriatr Med 2020;36:613-30.

7. Tavassoli N, Piau A, Berbon C, De Kerimel J, Lafont C, De Souto Barreto P, et al. Framework implementation of the INSPIRE ICOPE-CARE program in collaboration with the World Health Organization (WHO) in the Occitania Region. J Frailty Aging 2021;10:103-9.

8. Won CW, Lee Y, Lee S, Kim M. Development of Korean Frailty Index for Primary Care (KFI-PC) and its criterion validity. Ann Geriatr Med Res 2020;24:125-38.

9. Kim JA, Kim ES, Lee EK. Evaluation of the chronic disease management program for appropriateness of medication adherence and persistence in hypertension and type-2 diabetes patients in Korea. Medicine (Baltimore) 2017;96:e6577.

10. Kim H, Park YH, Jung YI, Choi H, Lee S, Kim GS, et al. Evaluation of a technology-enhanced integrated care model for frail older persons: protocol of the SPEC study, a stepped-wedge cluster randomized trial in nursing homes. BMC Geriatr 2017;17:88. 